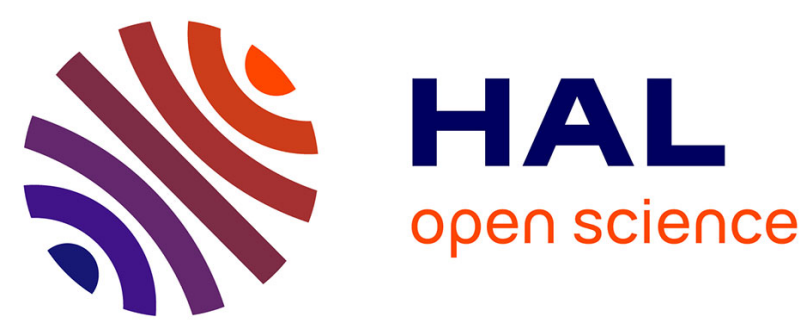

\title{
Statistical region-based active contours for segmentation: an overview
}

François Lecellier, Stéphanie Jehan-Besson, Jalal M. Fadili

\section{To cite this version:}

François Lecellier, Stéphanie Jehan-Besson, Jalal M. Fadili. Statistical region-based active contours for segmentation: an overview. Innovation and Research in BioMedical engineering, 2014, 35 (1), pp.3-10. 10.1016/j.irbm.2013.12.002 . hal-00918290

\section{HAL Id: hal-00918290 \\ https://hal.science/hal-00918290}

Submitted on 13 Mar 2014

HAL is a multi-disciplinary open access archive for the deposit and dissemination of scientific research documents, whether they are published or not. The documents may come from teaching and research institutions in France or abroad, or from public or private research centers.
L'archive ouverte pluridisciplinaire HAL, est destinée au dépôt et à la diffusion de documents scientifiques de niveau recherche, publiés ou non, émanant des établissements d'enseignement et de recherche français ou étrangers, des laboratoires publics ou privés.

\section{(ㅇ)(1) $\$$}

Distributed under a Creative Commons Attribution - NonCommercial - NoDerivatives| 4.0 


\title{
Statistical region-based active contours for segmentation: an overview
}

\author{
F. Lecellier ${ }^{\mathrm{a}}$, S. Jehan-Besson ${ }^{\mathrm{b}}$, J. Fadili ${ }^{\mathrm{b}}$ \\ ${ }^{a}$ XLIM-SIC, UMR CNRS 7252, Bd Marie et Pierre Curie BP 30179, 86962 Futuroscope \\ Chasseneuil cedex, France \\ ${ }^{b}$ GREYC CNRS-ENSICAEN-Université de Caen, UMR 6072, Bd du Maréchal Juin, 14050 \\ Caen, France
}

\begin{abstract}
In this paper we propose a brief survey on geometric variational approaches and more precisely on statistical region-based active contours for medical image segmentation. In these approaches, image features are considered as random variables whose distribution may be either parametric, and belongs to the exponential family, or non-parametric estimated with a kernel density method. Statistical region-based terms are listed and reviewed showing that these terms can depict a wide spectrum of segmentation problems. A shape prior can also be incorporated to the previous statistical terms. A discussion of some optimization schemes available to solve the variational problem is also provided. Examples on real medical images are given to illustrate some of the given criteria.
\end{abstract}

\section{Introduction}

Due to the rapid evolution of medical imaging systems, image segmentation faces various and increasingly difficult challenges. In order to cope with the growing variety of data encountered in medical imaging, segmentation tools must be able to handle different noise models and to provide a way to include geometric and photometric priors. To this end, geometric variational approaches that consider image segmentation as a continuous optimization problem are particularly well adapted (see for example [1-3]). Indeed, the computation of a given shape (i.e. segmentation) can be advantageously modeled as the optimum 
of a wisely chosen continuous energy functional. Continuous energy functionals allow the use of photometric image properties such as texture [4-8] and noise [9-12], as well as geometric properties such as the prior shape of the object to be segmented [13-18], see also [19] and more recently [20] for some reviews on deformable models and active contours in medical image segmentation.

In this paper, we propose to focus on region-based terms that take benefit of the probability density function of a given image feature inside the region of interest $[1,9,21]$. We more particularly review the optimization of divergences between pdfs which represent a general setting for both segmentation and tracking in medical images. When considering a segmentation framework, we aim at maximizing the distance between the pdf of the inside region and the pdf of the outside region. When considering a tracking application, we aim at minimizing the distance between the pdf of the region of interest and a reference one. The pdf can be considered as parametric (e.g. Gaussian, Rayleigh ...) or non parametric (no assumption is made on the law). In the literature, region tracking using non parametric probability density functions has been first proposed in [22] for video and then developed for cardiac structures tracking in perfusion MRI (p-MRI) sequences in [23]. On the other hand, some authors [24] have also proposed to take benefit of the maximization of the Bhattacharya distance of non parametric pdfs for segmentation. Concerning parametric pdfs, we restrict our study to the exponential family as first proposed in [9]. The rationale behind using the exponential family is that it includes, among others, Gaussian, Rayleigh, Poisson and Bernoulli distributions that have proven to be useful to model the noise structure in many real image acquisition devices (e.g. Poisson for photon counting devices such as X-ray or CCD cameras, Rayleigh for ultrasound images, etc). To cope with the occlusion and missing data and to alleviate initialization issues in medical image segmentation, a shape prior on the structure to be isolated can also prove necessary. For space reasons, we restrict our review to the use of the Legendre moments in a variational approach first proposed in [16] and applied to echocardiography in [25] and in cardiac segmentation on non-contrast CT images in [18]. 
One of the difficult point concerning variational approaches remains the associated resolution schemes. We propose here to review two main schemes. The first one is based on advanced and efficient optimization tools (e.g. originating from shape derivation tools [26] and the second one relies on efficient non-smooth convex optimization tools as proposed in [27]). Some segmentation examples of medical structures are taken (brain and cardiac MRI, echocardiography, MRI perfusion) to show the adaptability of such statistical segmentation methods.

\section{Statistical region-based terms for segmentation}

A region-based segmentation problem aims at finding a partition of the image domain $\Omega$ into $n$ regions $\left\{\Omega_{1}, . ., \Omega_{n}\right\}$ of respective boundaries $\left\{\partial \Omega_{1}, . ., \partial \Omega_{n}\right\}$ that minimizes the following criterion:

$$
E\left(\Omega_{1}, . ., \Omega_{n}, \Gamma\right)=\sum_{i=1}^{n} E_{r_{i}}\left(\Omega_{i}\right)+\lambda E_{b}(\Gamma),
$$

where $E_{r_{i}}$ is the region-based term related to the domain $\Omega_{i}$, and $\Gamma=\bigcup_{i=1}^{n} \partial \Omega_{i}$. The regularization term $E_{b}$ is balanced with a positive parameter $\lambda$.

We focus here on statistical region-based term $E_{r_{i}}$ that takes advantage of the pdf of some image features $\mathbf{y}$ whose realizations take values in $\chi \subset \mathbb{R}^{p}$ within each region $\Omega_{i}$ (e.g. when considering the intensity in grey level images $y(\mathbf{x})=I(\mathbf{x})$ with $\mathbf{x}$ the pixel location, $p=1$ and $\chi=[0,255])$. This framework is then also adapted to the use of vectorial image features such as the coefficients of the wavelet transform or optical flow vectors. At this stage, we consider two main classes of region descriptors detailed in the two sections below. We also provide a comparison for brain MRI segmentation in section 6.2.

\subsection{Statistical descriptors based on regions integrals}

For the first kind of region descriptors, we consider the minimization of region integrals of some well chosen functions of the pdf of the feature $\mathbf{y}$ within the region $\Omega_{i}$, namely $p\left(\mathbf{y}(\mathbf{x}), \Omega_{i}\right)$. We then consider as a region-based term:

$$
E_{r i}\left(\Omega_{i}\right)=\int_{\Omega_{i}} \Phi\left(p\left(\mathbf{y}(\mathbf{x}), \Omega_{i}\right) d \mathbf{x},\right.
$$


with $\Phi$ at least $\mathcal{C}^{1}$ and Lebesgue integrable function. Let us note that when $\Phi(t)=-\log (t)$ the function (2) is known as the log-likelihood score function, used to describe the homogeneity of a region. It has been introduced in [1] using Gaussian pdfs and in [9] using parametric pdfs from the exponential family. In [12], we consider a general setting replacing the - log by any proper function $\Phi$ within the exponential family. We also elucidate the impact of the estimation method of the hyper-parameters on the shape derivatives of the criterion. Such a criterion has been also investigated using non parametric pdfs and some functions $\Phi$ related to mutual information and entropy as developed in [28, 29].

\subsection{Statistical descriptors based on features integrals}

Another class of statistical region descriptors may be interesting since it can depicts both supervised and unsupervised segmentation. In this class of statistical descriptors, we consider integrals over the feature domain $\chi$ and so we do not make any assumption on the independence of the random variable $\mathbf{y}(\mathbf{x})$ on the contrary to the region descriptors described using (2). Let us then introduce the following functional which represents the distance, or more generally the divergence, between the current pdf estimate $p\left(\cdot, \Omega_{i}\right)$ and another one $q(\cdot)$ for some appropriate function $\Psi$ comparing pdfs:

$$
E_{r_{i}}\left(\Omega_{i}\right)=\int_{\chi} \Psi\left(p\left(\mathbf{y}, \Omega_{i}\right), q(\mathbf{y})\right) d \mathbf{y} .
$$

We can introduce for example the Hellinger distance or the commonly used Kullback-Leibler divergence. Some other distances or divergences can be introduced to improve the accuracy and robustness of the segmentation such as the Wasserstein distance [30] or alpha-divergences [31]. Segmentation of complex regions can also take benefit of local estimation of the pdfs on small patches as proposed in [32].

Such divergences represent a general setting for both segmentation and tracking in medical images. Indeed, we may cast the segmentation problem as the maximization of the distance between the pdfs of the feature in the inside and outside regions. In order to fix ideas, let us consider a partition of an image in 
two regions $\Omega_{1}$ and $\Omega_{2}$. The segmentation may be formulated as the maximization of the following criterion:

$$
E_{r}\left(\Omega_{1}, \Omega_{2}\right)=\int_{\chi} \Psi\left(p\left(\mathbf{y}, \Omega_{1}\right), q\left(\mathbf{y}, \Omega_{2}\right)\right) d \mathbf{y} .
$$

In the tracking problem, one aims at finding a consistent region $\Omega_{i}$ through a series of images. We can assume statistical similarity between the pdfs of the region in two consecutive images. We then search for the domain that minimizes the functional (3) where $q(\cdot)$ is a reference pdf that has been learned from the region of interest:

$$
E_{r_{i}}\left(\Omega_{i}\right)=\int_{\chi} \Psi\left(p\left(\mathbf{y}, \Omega_{i}\right), q_{r e f}(\mathbf{y})\right) d \mathbf{y} .
$$

This framework may also be applied to supervised segmentation where a reference pdf is learned on the region of interest.

\section{Statistical features description}

For all the above criteria, the pdf can be either parametric or non-parametric. Parametric pdfs are useful for the segmentation and tracking of homogeneous regions. The pdf can then be chosen according to the noise model as detailed thereafter. However parametric models may be subject to bias when the pdf model is only an approximation of the true noise model. In this case non parametric pdfs may be useful as well as in the case of segmentation or tracking of non homogeneous regions.

\subsection{Parametric pdfs within the exponential family}

In this case, image features are considered as random variables whose distribution belongs to some parametric family which is chosen according to the noise model. The exponential family is a flexible class of distributions that includes, among others, Gaussian, Rayleigh and Poisson distributions that have proven useful to model the noise structures in medical imaging systems. More formally, the current pdf estimate $p\left(\cdot, \Omega_{i}\right)$ is now parametrized by a vector $\boldsymbol{\theta}_{i} \in \Theta \subset \mathbb{R}^{\kappa}$ 
(e.g. we have $\kappa=2$ and $\boldsymbol{\theta}_{i}=\left(\mu_{i}, \sigma_{i}\right)^{T}$ where $\mu_{i}$ is the mean and $\sigma_{i}$ the variance for Gaussian law), or equivalently, by the natural parameter vector $\boldsymbol{\eta}_{i}$ in the terminology of the canonical exponential family [33]. The estimate of $\boldsymbol{\theta}_{i}$ (or $\boldsymbol{\eta}_{i}$ ) (performed using the maximum likelihood method for example) explicitly depends on the corresponding region, and this dependence must be taken into account when computing the derivatives of the functional. In [12], the expressions of the associated evolution equations were computed and detailed.

\subsection{Non-parametric pdfs}

Here we consider non-parametric density estimates using kernel-based methods. Given a region $\Omega_{i}$, we can estimate the pdf of the feature $y$ through the use of the Parzen method [34]. Let $K_{h}: \chi \rightarrow \mathbb{R}^{+}$be the Parzen window in general chosen as a Gaussian kernel of 0 -mean and variance $h$ (scale parameter):

$$
\hat{q}\left(\mathbf{y}, \Omega_{i}\right)=\frac{1}{\left|\Omega_{i}\right|} \int_{\Omega} K_{h}(\mathbf{y}(\mathbf{x})-\mathbf{y}) d \mathbf{x},
$$

where $y(\mathbf{x})$ is the value of the feature of interest at the point $\mathbf{x}$ of $\Omega_{i}$.

Such non parametric pdfs may be helpful for tracking non homogeneous regions (an example is given in section 6.3 for perfusion MRI) but also for the statistical description of more complex features such as the coefficients of linear texture transforms (e.g. wavelet, waveatoms). Some results are given in $[8,25]$.

\section{Segmentation with a shape prior}

Medical structures are often really complex and sometimes not very contrasted. The addition of a shape prior in the energy functional can then be crucial for many applications. Different approaches were considered that can be categorized as follows : minimization of a boundary-based functional [15]; shape prior through a signed distance function [13]; scale and translation invariant shape moments, e.g. Legendre as in [16].

In [17], we propose to combine statistical data term with a shape prior computed using its Legendre Moments. Indeed, moments [35] give a region-based 
compact representation of shapes through the projection of their characteristic functions on an orthogonal basis such as Legendre polynomials. Scale and translation invariance can be advantageously added as in [16]. When combining statistical region-based terms and shape prior term, the criterion becomes:

$$
E\left(\Omega_{\text {in }}, \Omega_{\text {out }}, \Gamma\right)=E_{\text {in }}\left(\mathbf{x}, \Omega_{\text {in }}\right)+E_{\text {out }}\left(\mathbf{x}, \Omega_{\text {out }}\right)+\alpha D\left(\Omega_{\text {in }}, \Omega_{\text {ref }}\right)+\beta E_{b}(\Gamma),
$$

where $D\left(\Omega_{i n}, \Omega_{r e f}\right)$ is defined as the Euclidian distance between the moments of the evolving region and the ones of the reference shape:

$$
D\left(\Omega_{i n}, \Omega_{r e f}\right)=\left\|\lambda\left(\Omega_{i n}\right)-\lambda\left(\Omega_{r e f}\right)\right\|_{2}^{2},
$$

where $\lambda\left(\Omega_{i}\right)$ are the moments of the region $\Omega_{i}$.

To drive this functional towards its minimum, the obtained evolution equation is iteratively run without the shape prior, then the shape prior term is updated, and the active contour evolves again by running the PDE with the shape prior. This procedure is repeated until convergence. An example of the segmentation of the left ventricle in echocardiography is given in section 6.1.

\section{Resolution schemes for active contour segmentation}

The statistical criteria introduced above are generic and allow to well describe numerous medical image segmentation problems. However they imply the estimation of the optimal domain using an appropriate resolution method. This is the key part in variational approaches and we here propose to focus on two main efficient methods to solve the previous optimization problems.

\subsection{Methods based on the evolution of a deformable model}

These methods are based on the computation of an evolution equation that will drive an active shape towards the boundary of the object to segment. Such a deformation is directed by a geometric partial differential equation (PDE). Let us denote by $\Gamma(s, \tau)$ the active contour where $s$ is a parameter of the contour and $\tau$ is an evolution parameter. The contour evolves according to the following 
well known PDE: $\frac{\partial \Gamma(s, \tau)}{\partial \tau}=F \mathbf{N}$ where $\mathbf{N}$ is the normal vector and the velocity $F$ must be deduced from each considered criterion.

In order to perform the computation of this PDE, several methods have been introduced. The first one is the well-known approach proposed in [2], which consists in transforming the initial criterion by introducing the level set function $U$ and computing the associated Euler-Lagrange equations. The second one has been proposed by [36] and takes advantage of shape derivative tools introduced in [26]. Let us suppose that the shape derivative of the criterion $E(\Omega)$ in the direction $\mathbf{V}$ may be written as follows:

$$
<E^{\prime}(\Omega), \mathbf{V}>=-\int_{\partial \Omega} \operatorname{speed}(\mathbf{x}, \Omega)(\mathbf{V}(\mathbf{x}) \cdot \mathbf{N}(\mathbf{x})) d \mathbf{a}(\mathbf{x}) .
$$

If we want to minimize $E$, we can choose $F=\operatorname{speed}(\mathbf{x}, \Omega)$. On the contrary, to maximize the criterion, we take the opposite sign for the velocity.

Some other approaches are also worth mentioning. For example, some authors propose to directly take benefit of B-splines in the level set formulation [37] allowing efficient resolution schemes, or to implement fast Newton-type minimization schemes based on second order derivatives in [38].

\subsection{Convexification method}

This approach was proposed [27] for the classical non-convex variational segmentation problem:

$$
\min _{\Omega_{1}} E=\int_{\Omega_{1}}\left(I(\mathbf{x})-c_{1}\right)^{2} d \mathbf{x}+\int_{\Omega \backslash \Omega_{1}}\left(I(\mathbf{x})-c_{2}\right)^{2}+\lambda \int_{\partial \Omega_{1}} g(\mathbf{x}) d \mathbf{a}(\mathbf{x}),
$$

where $c_{i}$ is the mean value of the intensity within the corresponding region, and $g(\cdot)$ is some scalar function on the boundary.

The key idea now is (i) to reformulate this functional by introducing the characteristic function $u$ of $\Omega_{1}$; (ii) to use the co-area formula to re-express the boundary integral in terms of the TV semi-norm of $u$; and (iii) to relax $u$ to live in $[0,1]$. Altogether, this yields the non-smooth but convex functional:

$\min _{0 \leq u \leq 1} E_{G M}=\int_{\Omega}\left(u(\mathbf{x})\left(\mathbf{y}(\mathbf{x})-c_{1}\right)^{2}+(1-u(\mathbf{x}))\left(\mathbf{y}(\mathbf{x})-c_{2}\right)^{2}+\lambda g(\mathbf{x})|\nabla u(\mathbf{x})|\right) d \mathbf{x}$. 


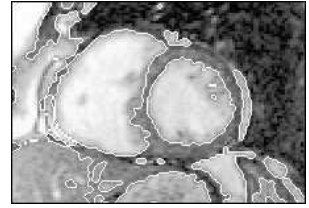

(a)

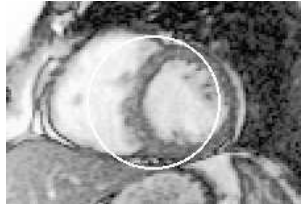

(b)

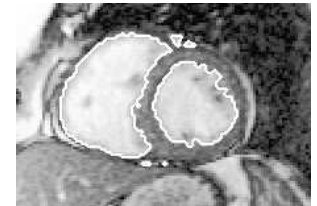

(c)

Figure 1: Segmentation of a cardiac MRI slice obtained using the convexification approach [27] (a) and active contours [2] (c) (using the initial contour given in (b)).

In [39], the authors show that, for fixed $c_{1}$ and $c_{2}$, if $u^{*}$ is a global minimizer of $E_{G M}$, then for almost every $\alpha \in[0,1], \Omega_{C}(\alpha):=\left\{\mathbf{x} \in \Omega: u^{*}(\mathbf{x})>\alpha\right\}$ is a global minimizer of $E$. The energy $E_{G M}$ can be solved using efficient nonsmooth convex optimization tools as proposed in [27].

In Fig. 1, we give a comparison between active contours and the convexification approach from [27] for a MRI slice of the 2009 MICCAI challenge database [40] . Using active contours, and given that the energy $E$ is non-convex, the final contour Fig. 1.(c) depends on the choice of the initial contour of Fig. 1.(b), which is only a stationary point (not necessarily a minimizer even local). Using the convexification approach, the obtained $u^{*}$ is indeed a global minimizer, but must be thresholded using the parameter $\alpha$. One of the advantage of convex optimization tools is the low computational cost of its resolution schemes. The introduction of more complex descriptors is also possible and an interesting application was proposed in [18] for cardiac segmentation with a prior shape based on Legendre moments.

\section{Medical image segmentation examples}

In this section we present some results on medical image segmentation using the criteria introduced in section 2. We propose different examples using both parametric and non parametric pdfs for different applications: echocardiography, brain MRI and perfusion MRI. 


\subsection{Noise and shape prior for cardiac echography segmentation}

We propose to illustrate the interest of using shape prior on real echocardiographic images. We use a parametric Rayleigh model for the noise distribution with an anti-log-likelihood homogeneity criterion, and a shape prior term with invariant Legendre moments and an alternating minimization scheme. In order to deal with the shape variability of the heart on images, we choose $\alpha=0.7$ and $\beta=0.4$ on eq. (6). Those parameters give the best results on quantitative and qualitative evaluation of the segmentation as shown in [25].

We conduct our experiment on both parietal and apical views and on contrast and classical echocardiography. The results on apical view for classical echography are depicted in Fig. 2. The importance of adding a shape prior in echocardiographic data is here demonstrated. In addition to this comparison, we compare also this method to another well-known segmentation method, the Active Appearance and Motion Models (AAMM) proposed by Bosch [41]. In Fig. 2 (d), we present the segmentation result of the echographic image using AAMM. We also compute the mean distance between AAMM and expert segmentation (cross points on Fig. 2 (e)), and the mean distance between active contours and expert segmentation (circle points on Fig. 2 (e)). As depicted on Fig. 2 (e), active contours are closer to expert segmentation than AAMM. In addition, we compute Dice coefficient for both methods, on AAMM it is 0.74 and on active contour it is 0.83 . Further quantitative evaluation results may be found in [25].

\subsection{Parametric pdfs for brain MRI segmentation}

Here we give an example on simulated brain T1-weighted MRI images provided by the Montreal Neurological Institute Brain Web URL. We perform the segmentation of the WM on a brain MRI image with a noise of $7 \%$ (Brain Web offers noise levels ranging from 1 to $9 \%$ ). For such images the problem of segmentation is particularly critical for both diagnosis and treatment purposes. It becomes necessary to obtain a robust segmentation of the different tissues (WM (White Matter), GM (Gray Matter), or Cerebrospinal Fluid (CSF)) or 


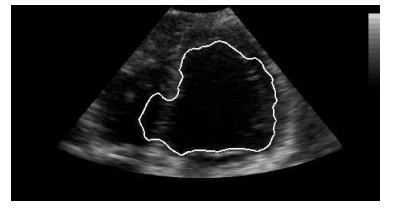

(a)

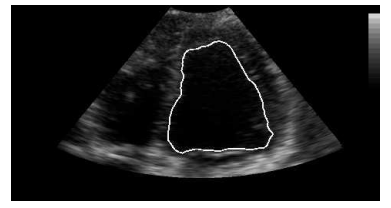

(b)

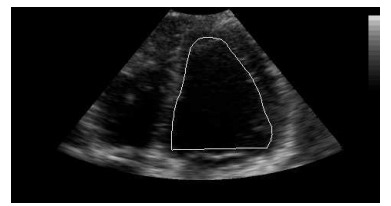

(c)

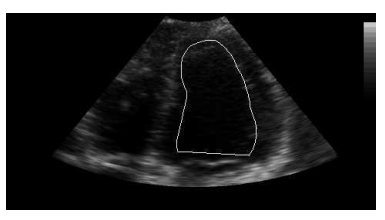

(d)

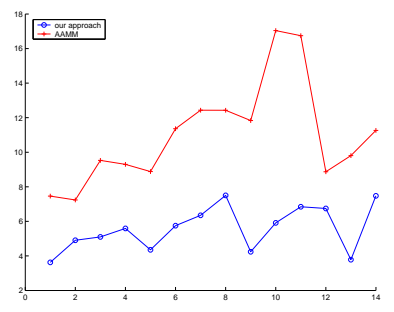

(e)

Figure 2: Segmentation of the left ventricle in echocardiography using a Rayleigh model of the noise without any shape prior (a) and with a shape prior (b). The expert contour is given in (c) and the segmentation result of AAMM method [41] is given in (d). The graphic (e) gives the mean distance computed on 14 images between the AAMM and expert (cross points) and between the shape and noise method and expert (circle points).

structures. The noise model in MRI images is assumed to be represented by a Rician distribution. However, for large signal intensities the noise distribution can be considered as a Gaussian distribution (this is the case for the WM or the GM). For the CSF which has a low signal intensity, the noise model can be approximated by a Rayleigh noise.

In Fig. 3, we first compare the Chan-Vese method [2] to the (anti-)loglikelihood with Gaussian noise [9] and divergence maximization using Kullback Leibler (KL) for the WM segmentation. We can note that the KL criterion acts as an extractor of the most prominent Gaussian in the initial mixture of Gaussians. The two other criteria separate the mixture without extracting a single Gaussian. In order to extract single Gaussian pdfs using the Chan-Vese method, a multiphase procedure can be advantageously used (see [42]). From a quantitative point of view, our method gives a dice coefficient of 0.91 , a False Positive Fraction (FPF) of $0.8 \%$ and a True Positive Fraction (TPF) of $84 \%$ (computed on the whole volume). We can remark that our method gives a 


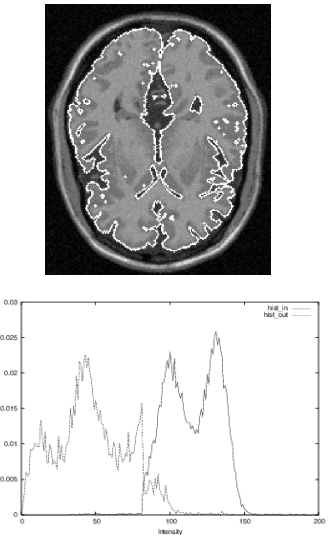

(a) Chan \& Vese
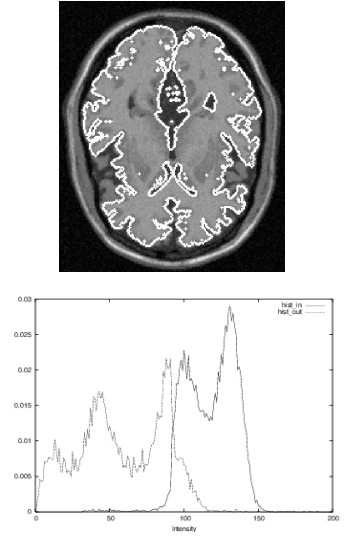

(b) $\log$ likelihood
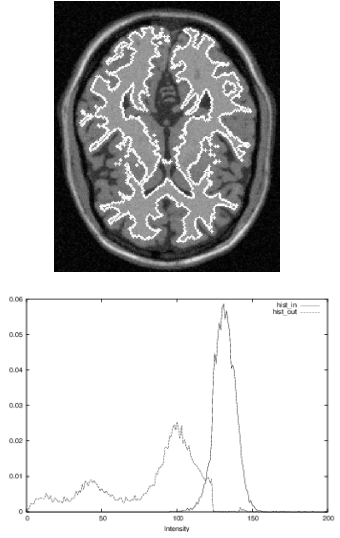

(c) KL maximization

Figure 3: T1-weighted brain MRI segmentation results (extraction of the White Matter). Column (a): final contour and pdfs for the Chan \& Vese method [2], column (b): for the log-likelihood method [1], column (c): for the maximization of the KL divergence.

very small number of false positive voxels, at the price of a higher number of missing voxels. The last point is due to the regularization term which acts by minimizing the curve length. Some complementary results are depicted in [43].

\subsection{Non parametric pdfs for perfusion MRI tracking}

The perfusion MRI (p-MRI) has emerged as a primordial clinical investigation tool in the evaluation of cardiac diseases. Spatio-temporal tracking of myocardial dynamics during the first transit of the contrast bolus allows the identification of hypoperfused or ischemic regions. An automatic quantification tool relies on the accurate segmentation and tracking of the cardiac structures. The main difficulty lies in the fact that the different regions (myocardium, left ventricle) are not homogeneous. We give here an example of tracking using non parametric pdfs. From an initial segmentation of the left ventricle, we track this structure along the sequence by minimizing the distance between the pdf of the intensity of the current region and the pdf of the intensity of the previous one. In Figure 4, we show the evolution of the curve in one frame of the sequence and the joint evolution of the pdf of the inside region. It is interesting to see 
how the pdf converges towards the reference pdf as shown in Figure 4. This example shows that this tool can be used for segmentation tracking but some improvements are needed notably concerning the fact that the criterion needs both the inside reference pdf and an outside reference one. The outside reference pdf can be for example computed on the narrowband as proposed in [44] in order to be more representative.

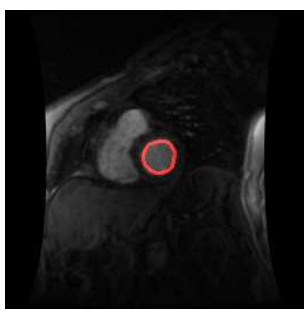

(a) reference segmentation

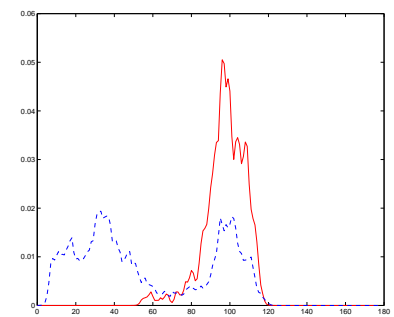

(d) initial pdfs (initial contour)

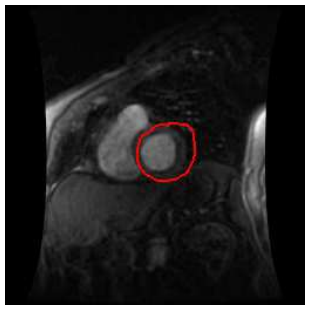

(b) initial contour

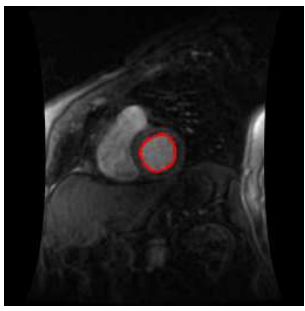

(c) final contour

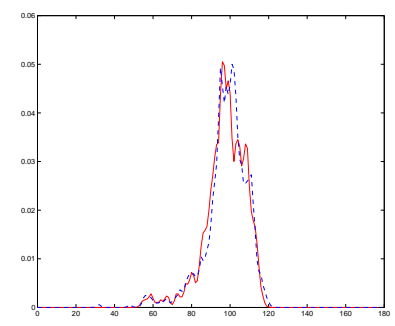

(g) final pdfs (final contour)

Figure 4: Minimization of the distance between the current region pdf (blue or dashed line) and a reference one (red or solid line) which is computed from the segmentation of LV in the previous frame (the pdf is the pdf of the intensity $I$ within the region).

\subsection{Discussion}

Experimental results are given in order to prove the applicability of our general setting to medical image segmentation. Indeed, we can observe that various types of noise often contribute to degrade medical images (Gaussian, Poisson, Rayleigh). Our general framework allows the use of the noise models that are recovered by the exponential family. As studied in [12], the noise model has an influence on the accuracy of the segmentation and on its robustness to the 
choice of the regularization parameter. The examples show that relevant result are obtained both in MRI and echocardiographic images. When dealing with non homogeneous objects, we propose to rather take benefit of non parametric pdfs where no assumption is made on the distribution model. This has been exploited for tracking the LV in p-MRI sequences. Concerning parametric pdfs, the parameters of the noise models depend on the regions inside and outside the active contours and they are estimated automatically during the segmentation process using the ML (maximum likelihood) estimator. The only parameter to set is then the regularization parameter $\lambda$ that allows to smooth the contour. Setting this parameter too high may lead to an under-segmentation of the structure and so its value is taken around 10 and 100 for the experimental results given above. However, experiments reported in [25] show that choosing the appropriate noise model allows the segmentation to be less sensitive to the choice of this parameter. As far as non parametric pdfs are concerned, the kernel parameter $h$ allows to smooth the initial pdf. However, we set this value to 1 because we want an estimate of the pdf very close to the initial pdf for the accuracy of the tracking.

Let us mention that a recent trend in medical image segmentation consists in using the computed velocity of the active contour as a basis for an interactive segmentation tool, see for instance [45]. The tools reviewed above can then be exploited not only for automatic segmentation but also as a basis for an interactive segmentation process to guide the practitioner.

\section{$7 \quad$ Acknowledgements}

We would like to thank Dr. E. Saloux (CHU of Caen) for providing the echocardiographic images and Pr. M. Hamon (CHU Caen) for providing the perfusion MRI images. We also thank D. Tschumperlé and R. Clouard from GREYC Laboratory for their useful image processing libraries Gmic and Pandore.

[1] S. Zhu, A. Yuille, Region competition: unifying snakes, region growing, and bayes/MDL for multiband image segmentation, IEEE PAMI 18 (1996) 884-900. 
[2] T. Chan, L. Vese, Active contours without edges, IEEE TIP 10 (2) (2001) 266277.

[3] E. Debreuve, M. Barlaud, G. Aubert, I. Laurette, J. Darcourt, Space-time segmentation using level set active contours applied to myocardial gated SPECT, IEEE TMI 20 (7) (2001) 643-659.

[4] N. Paragios, R. Deriche, Geodesic active regions and level set methods for supervised texture segmentation, IJCV 46 (3) (2002) 223-247.

[5] J.-F. Aujol, G. Aubert, L. Blanc-Féraud, Wavelet-based level set evolution for classification of textured images, IEEE TIP 12 (12) (2003) 1634-1641.

[6] D. Cremers, M. Rousson, R. Deriche, A review of statistical approaches to level set segmentation: integrating color, texture, motion and shape, IJCV 72 (2007) $195-215$.

[7] I. Karoui, R. Fablet, J. M. Boucher, J. M. Augustin, Region-based image segmentation using texture statistics and level-set methods, in: ICASSP, Vol. 2, 2006, pp. 693-696.

[8] J. Fadili, F. Lecellier, S. Jehan-Besson, Multiscale transforms for region-based texture segmentation, in: SAMPTA, Singapore, 2011, p. P0175.

[9] P. Martin, P. Réfrégier, F. Goudail, F. Guérault, Influence of the noise model on level set active contour segmentation, IEEE PAMI 26 (6) (2004) 799-803.

[10] F. Lecellier, S. Jehan-Besson, J. Fadili, G. Aubert, M. Revenu, Statistical regionbased active contours with exponential family observations, in: ICASSP, Vol. 2, 2006, pp. 113-116.

[11] F. Galland, N. Bertaux, P. Réfrégier, Multi-component image segmentation in homogeneous regions based on description length minimization: Application to speckle, Poisson and Bernoulli noise, Pattern Recognition 38 (2005) 1926-1936.

[12] F. Lecellier, J. Fadili, S. Jehan-Besson, G. Aubert, M. Revenu, E. Saloux, Regionbased active contours with exponential family observations, JMIV 36 (2010) 2845 . 
[13] M. Leventon, Statistical models for medical image analysis, Ph.D. thesis, Harvard (2000).

[14] A. Tsai, A. Yezzi, W. Wells, A shape-based approach to the segmentation of medical imagery using level sets, IEEE TMI 22 (2003) 137-154.

[15] M. Gastaud, M. Barlaud, G. Aubert, Combining shape prior and statistical features for active contour segmentation, IEEE TCSVT 14 (5) (2004) 726-734.

[16] A. Foulonneau, P. Charbonnier, F. Heitz, Affine-invariant geometric shape priors for region-based active contours, IEEE PAMI 28 (2006) 1352-1357.

[17] F. Lecellier, S. Jehan-Besson, J. Fadili, G. Aubert, M. Revenu, E. Saloux, Regionbased active contours with noise and shape priors, in: ICIP, Vol. 1, 2006, pp. $1649-1652$.

[18] J. Wojak, E. D. Angelini, I. Bloch, Introducing shape constraint via Legendre moments in a variational framework for cardiac segmentation on non-contrast CT images, in: VISAPP, 2010, pp. 209-214.

[19] N. Paragios, J. Duncan, N. Ayache, Biomedical Image Analysis: Methodologies and Applications, Springer, 2011.

[20] O. Bernard, P. Clarysse, T. Dietenbeck, D. Friboulet, S. Jehan-Besson, J.Pousin, Analyse d'images cardiaques et thoraciques, IC2-Information et Science du Vivant Hermes-Lavoisier, 2014, Ch. Extraction et segmentation de structures dans des séquences d'images, p. to appear.

[21] N. Paragios, R. Deriche, Geodesic active regions: A new paradigm to deal with frame partition problems in computer vision, JVCIR 13 (2002) 249-268.

[22] G. Aubert, M. Barlaud, O. Faugeras, S. Jehan-Besson, Image segmentation using active contours : Calculus of variations or shape gradients, SIAM Applied Mathematics 63 (2003) 2128-2154.

[23] N. Rougon, C. Petitjean, F.Preteux, P.Cluzel, P.Grenier, A non-rigid registration approach for quantifying myocardial contraction in tagged MRI using generalized information measures, MIA (2005) 353-375. 
[24] O. Michailovich, Y. Rathi, A. Tannenbaum, Image segmentation using active contours driven by the Bhattacharyya gradient flow, IEEE TIP 16 (2007) 2787 2801.

[25] F. Lecellier, Les contours actifs basés région avec a priori de bruit, de texture et de forme : Application à l'échocardiographie, Ph.D. thesis, University of Caen, France (2009).

[26] M. Delfour, J. Zolésio, Shape and geometries, Advances in Design and Control, SIAM, 2001.

[27] X. Bresson, S. Esedoglu, P. Vandergheynst, J. Thiran, S. Osher, Fast global minimization of the active contour/snake model, Journal of Mathematical Imaging and Vision 28 (2007) 151-167.

[28] J. Kim, J. Fisher III, A. Yezzi Jr., M. Cetin, A. Willsky, Nonparametric methods for image segmentation using information theory and curve evolution, in: ICIP, 2002, pp. 797-800.

[29] A. Herbulot, S. Jehan-Besson, M. Barlaud, G. Aubert, Shape gradient for multimodal image segmentation using mutual information, in: ICIP, Singapore, 2004, pp. 2729-2732.

[30] G. Peyré, J. Fadili, J. Rabin, Wasserstein active contours, in: ICIP, 2012, pp. $2541-2544$.

[31] L. Meziou, A. Histace, F. Precioso, Alpha-divergence maximization for statistical region-based active contour segmentation with non-parametric pdf estimations, in: ICASSP, 2012, pp. 861-864.

[32] N. Kangyu, X. Bresson, T. Chan, S. Esedoglu, Local histogram based segmentation using the wasserstein distance, IJCV 84 (1) (2009) 97-111.

[33] P. Bickel, K. Docksum, Mathematical statistics: basic ideas and selected topics, 2nd Edition, Vol. I, Prentice-Hall, London, 2001.

[34] R. Duda, P. Hart, Pattern Classification and Scene Analysis, John Wiley and Sons, Inc., 1973. 
[35] C. H. The, R. T. Chin, On image analysis by the methods of moments, IEEE PAMI 10 (1988) 496-513.

[36] S. Jehan-Besson, M. Barlaud, G. Aubert, DREAM2S: Deformable regions driven by an eulerian accurate minimization method for image and video segmentation, IJCV 53 (1) (2003) 45-70.

[37] O. Bernard, D. Friboulet, P. Thevenaz, M. Unser, Variational B-spline level-set: A linear filtering approach for fast deformable model evolution, IEEE TIP 18 (6) (2009) 1179-1191.

[38] L. Bar, G. Sapiro, Generalized newton-type methods for energy formulations in image processing, SIAM J. Imaging Sci. 2 (2009) 508-531.

[39] M. Nikolova, S. Esedoglu, T. F. Chan, Algorithms for finding global minimizers of image segmentation and denoising models, SIAP 66 (5) (2006) 1632-1648.

[40] P. Radau, Y. Lu, K. Connelly, G. Paul, A. Dick, G. Wright, Evaluation framework for algorithm segmenting short axis cardiac MRI, The MIDAS Journal Cardiac MR Left Ventricle Segmentation Challenge (2009).

[41] J. G. Bosch, S. C. Mitchell, B. P. F. Lelieveldt, F. Nijland, O. Kamp, M. Sonka, J. H. C. Reiber, Automatic segmentation of echocardiographic sequences by active appearance motion models, IEEE TMI 21 (2002) 1374-1383.

[42] E. Angelini, T. Song, B. Mensh, A. Laine, Brain MRI segmentation with multiphase minimal partitioning: a comparative study, Int. J. of Biomedical Imaging (2007) 15 p. ID 10526.

[43] S. Jehan-Besson, F. Lecellier, J. Fadili, G. Nee, G. Aubert, Biomedical diagnostics and clinical technologies : Applying high performance cluster and Grid Computing, IGI global, 2011, Ch. Medical image segmentation and tracking through the maximisation or the minimisation of divergences between pdfs, pp. 34-61.

[44] J. Mille, J.-L. Rose, Region tracking with narrow perception of background, in: ICIP, 2011, pp. 493-496.

[45] K. Bianchi, A. Vacavant, R. Strand, P. Terv, L. Sarry, Dual B-spline snake for interactive myocardial segmentation, in: Int. Conf. on Medical Image Understanding and Analysis, Birmingham, UK, 2013, pp. 131-136. 
